(ECs). Our objective was to determine whether physiological concentrations of ADMA had a positive effect on NO production via CaSR stimulation.

Methods Inducible CaSR overexpressing HEK293 cells (HekCaSR) were generated using the FLP-IN T-REX transfection method. Intracellular $\mathrm{Ca} 2+$ mobilisation was assessed using the $\mathrm{Ca} 2+$ sensitive dye CAL520, while cAMP accumulation was measured using an ELISA kit. CaSR was stimulated by $\mathrm{Gd} 3+$ in the presence of $10 \mu \mathrm{M}$ ADMA. ADMA-CaSR signalling in ECs was investigated using human lung microvascular ECs (HuLMVECs). NO production was assessed using the fluorescent sensor DAF-AM. The CaSR antagonist NPS-2143 was used to show specificity to CaSR signalling.

Results $\mathrm{Gd} 3+$ releases stored intracellular $\mathrm{Ca} 2+$ and suppresses cAMP production in a dose dependent manner in HEK-CaSR cells. Incubation with $10 \mu \mathrm{M}$ ADMA for 1 hour prior to $\mathrm{Gd} 3$ + stimulation was found to sensitise CaSR and shift the dose-response curve for $\mathrm{Ca} 2+$ mobilisation leftward (CTRL EC50 0.16mM, ADMA EC50 $0.05 \mathrm{mM}$ p=0.01). ADMA pre-treatment suppressed forskolin induced cAMP production to $70 \%$ of the maximal response. $\mathrm{Ca} 2+$ stimulation of HuLMVECs releases intracellular $\mathrm{Ca} 2+$ in a dose dependent manner, in the presence of $10 \mu \mathrm{M}$ ADMA maximal fluorescence is increased with no change to the EC50. Given cytosolic $\mathrm{Ca} 2+$ release increases NOS activity we determined whether low concentrations of ADMA $(10 \mu \mathrm{M})$ promoted NO production. At maximal CaSR stimulation ADMA increased NO production by $40 \%(\mathrm{p}=0.04)$. Consistent with previous reports higher concentrations of ADMA $(100 \mu \mathrm{M})$ blocked NO production through inhibition of NOS. The action of
ADMA was blocked by the addition of the CaSR inhibitor NPS-2143.

Conclusion Physiological concentrations of ADMA increase CaSR-mediated intracellular $\mathrm{Ca} 2+$ release and increase CaSRmediated suppression of cAMP synthesis. In ECs, physiological concentrations of ADMA increase $\mathrm{Ca} 2+$ stimulated $\mathrm{NO}$ synthesis. In contrast pathophysiological concentrations directly inhibit EC NO synthase. These data suggest that physiological concentrations of ADMA positively regulate vascular reactivity while high concentrations of ADMA seen in cardiovascular disease reduce vascular function through NO blockade.

Conflict of interest None

\section{BS42 HOW WARFARIN AND ANTIPLATELETS AFFECT CLOT STRUCTURE IN ATRIAL FIBRILLATION}

${ }^{1}$ Ahsan Khan* ${ }^{2}{ }^{2}$ Eduard Shantsila, ${ }^{2}$ Y.C. Lau, ${ }^{3}$ Lewis Hardy, ${ }^{3}$ Helen Philippou, ${ }^{1}$ Gregory Lip. ${ }^{1}$ Liverpool Heart \& Chest Hospital NHS Foundation Trust; ${ }^{2}$ University of Birmingham; ${ }^{3}$ University of Leeds

\subsection{6/heartjnl-2019-BCS.204}

Introduction Atrial fibrillation (AF) and coronary artery disease (CAD) are two common cardiovascular conditions associated with increased morbidity and mortality. Both conditions bring a risk of thrombosis and thus antithrombotic therapy is indicated. Despite recognised treatment, stroke and myocardial infarctions still occur. Accordingly, the identification of patients whose haemostasis remains unimpaired by treatment is valuable.

Abstract BS42 Table 1 Demographic, clinical and heamostatic characteristic of patients with atrial fibrillation and coronary artery disease (control group)

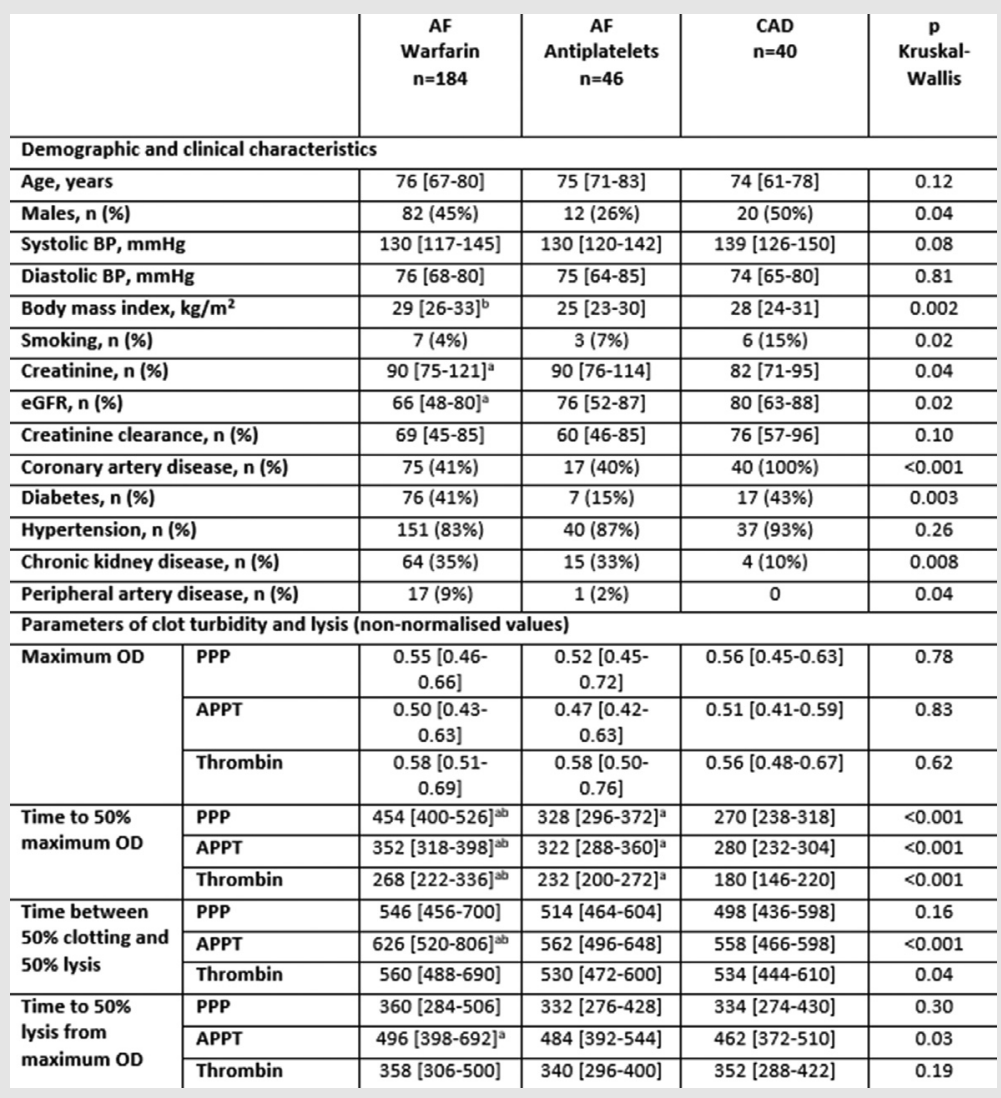


Purpose To investigate differences in plasma clot properties in patients with $\mathrm{AF}$ and $\mathrm{CAD}$ and compare the effect of warfarin and antiplatelets on clot structure in AF population.

Methods We studied 270 patients and divided them into 3 groups: AF on warfarin $(n=184)$, AF on antiplatelets $(n=46)$ and CAD $(n=40)$. Plasma samples were obtained from participants and centrifuged to prepare platelet poor plasma. Assays were performed in 96-well polystyrene microtiter plates. Reagents were diluted in standard buffer $(10 \mathrm{mM} \mathrm{N}$-2-hydroxyethylpiperazine-N'-2-ethanesulphonic acid [HEPES], $\mathrm{pH}$ 7.4, $150 \mathrm{mM} \mathrm{NaCl})$. Patient plasma samples $(25 \%)$ were incubated with tissue plasminogen activator (500 ng.mL-1) for 10 minutes at $37 \hat{\mathrm{EC}}$ before the addition of $\mathrm{CaCl} 2(7.5 \mathrm{mM})$. Either PPP reagent $(2.5 \%)$, aPPT reagent $(2.5 \%)$, or thrombin $(0.5$ U.mL-1) were then added to initiate coagulation. Polymerisation of fibrin in plasma was monitored ( $\triangle$ OD340 nm) using a Synergy $\mathrm{H} 1$ hybrid multi-mode plate reader, readings were taken in 12 second intervals for up to 60 minutes.

Results Comparisons between the 3 groups was performed using Kruskal-Wallis test, with Dunn's post-hoc analysis and Holm-Sidak adjustment. There were no significant differences in clot structure between 3 subgroups. The maximum rate of clot formation was significantly delayed in the warfarin subgroup with all reagents used $(\mathrm{p}<0.001)$ (table 1$)$. Plasma clot susceptibility to fibrinolysis increased with warfarin compared to antiplatelets but was significant only with APPT and thrombin reagents $(\mathrm{p}<0.001$ and 0.04 respectively).

Conclusion Warfarin was effective in delaying clot formation compared to antiplatelets and also resulted in increased susceptibility of plasma clot to fibrinolysis.

Conflict of interest None

\section{BS43 ABSTRACT WITHDRAWN}

\section{BS44 CYTOKINE INDUCED DOWNREGULATION OF PLASMA MEMBRANE CALCIUM ATPASE 4 GENE INCREASES SENSITIVITY TO APOPTOSIS IN PULMONARY ARTERY ENDOTHELIAL CELLS}

\begin{abstract}
${ }^{1}$ Jude Ihugba*, ${ }^{2}$ Satishkumar Kurusamy, ${ }^{2}$ Reshma Naomi Ranjit Immanuel, ${ }^{2}$ Kinza Khan, ${ }^{2}$ Jayashree Jayachandran, ${ }^{3}$ Nadine Arnold, ${ }^{2}$ Priscille Polla, ${ }^{4}$ Pablo Gomez-del Arco, ${ }^{5}$ Juan Miguel Redondo, ${ }^{6}$ James Cotton, ${ }^{7}$ Paul D Upton, ${ }^{8}$ Nicholas Morrell, ${ }^{3}$ Allan Lawrie, ${ }^{2}$ Angel $\mathrm{L}$ Armesilla. ${ }^{1}$ Research Institute in Healthcare Science; ${ }^{2}$ RIHS, FSE, University of Wolverhampton; ${ }^{3}$ Department of Infection, Immunity and Cardiovascular Disease, University of Sheffield, Sheffield, UK; ${ }^{4}$ IIER, Instituto de Salud Carlos III, Madrid Spain; ${ }^{5}$ Centro Nacional de Investigaciones Cardiovasculares, Madrid, Spain; ${ }^{6}$ Department of Cardiology, Heart and Lung Centre, New Cross Hospital, Wolverhampton, UK; ${ }^{7}$ Department of Medicine, University of Cambridge, Addenbrooke's and Papworth Hospitals, Cambridge, UK; ${ }^{8}$ University of Cambridge
\end{abstract}

10.1136/heartjnl-2019-BCS.205

Introduction Pulmonary arterial hypertension (PAH) is a lifethreatening disease characterized by progressive vasoconstriction, vascular remodelling, and occlusion of small pulmonary arteries. It leads to increased pulmonary resistance and finally right ventricular failure. The disorder has no cure and current therapies only target vasoconstriction but have little effect on vessel remodelling. Increased activity of pro-inflammatory cytokines is linked to $\mathrm{PAH}$ pathogenesis. In this study, we analysed the effect of TNF-alpha and IL-1Betaßon the expression of Plasma Membrane Calcium ATPase 4 (PMCA4) in pulmonary artery endothelial cells (PAEC).
Methods PAEC were cultured for different times and with different doses of TNF-alpha or IL-1Beta. Expression of PMCA4 RNA and protein was determined by qPCR and western blot respectively. PMCA4 expression was silenced using siRNA specific for human PMCA4. Quantification of apoptotic cells was performed by flow cytometry and TUNNEL.

Results Treatment of PAEC with TNF-alpha or IL-1Beta induced a time- and dose-dependent decrease in the levels of RNA for PMCA4. Analysis of PMCA4 RNA levels in the lungs of mice with overexpression of ectopic TNF-alpha confirmed the in vivo relevance of these observations. RNA decay experiments performed by blocking cellular transcription with Actinomycin D indicate that the downregulation of PMCA4 RNA levels mediated by pro-inflammatory stimuli in PAEC is the result of a decrease in RNA stability. In agreement with the reduction observed in RNA levels, PMCA4 protein expression was strongly decreased by treating PAEC with TNF-alpha or IL-1Beta. Silencing PMCA4 gene expression sensitised PAEC to apoptosis, suggesting that PMCA4 protects PAEC to apoptosis induced by pro-inflammatory cytokines.

Conclusion The pro-inflammatory cytokines TNF-alpha and IL-1Beta significantly downregulate the expression of the PMCA4 gene in PAEC at the RNA and protein level. Decrease in PMCA4 expression sensitised PAEC to apoptosis. This indicates that the PMCA 4 gene might play an important role in the apoptotic loss of endothelial cells observed in the pulmonary arterioles of patients with $\mathrm{PAH}$.

Conflict of interest None

\section{BS45 ACTIVATING TRANSCRIPTION FACTOR ATF2 NEGATIVELY REGULATES THE EXPRESSION OF ENDOTHELIAL NOTCH LIGANDS}

${ }^{1}$ Ivonne Olivares*, ${ }^{1}$ Krithika Kalyanakrishnan, ${ }^{1}$ Suhail Ahmed, ${ }^{1}$ Clare Murcott, ${ }^{2}$ Robert $\mathrm{N}$ Wilkinson, ${ }^{3}$ James Cotton, ${ }^{4}$ Wolfgang Breitwieser, ${ }^{1}$ Mark R Morris, ${ }^{1}$ Angel L Armesilla. ${ }^{1}$ RIHS, FSE, University of Wolverhampton; '2Department of Infection, Immunity \& Cardiovascular Disease \& Bateson Centre, University of Sheffield; ${ }^{3}$ Department of Cardiology, Heart and Lung Centre, New Cross Hospital, Wolverhampton, UK; ${ }^{4} 5$ Molecular Biology Core Facility, Cancer Research UK, Manchester, UK

\subsection{6/heartjnl-2019-BCS.206}

Introduction ATF2 also known as cyclic AMP response element binding protein 2 (CREB2) is a member of the leucine zipper (bZIP) transcription factor family that binds to specific DNA sequences and regulates the transcriptional activation of target genes. ATF2 regulation and functions have been studied in a number of developmental and pathological conditions. Studies have shown that activation of ATF2 by VEGF mediates angiogenic processes such as endothelial cell migration and tubular morphogenesis but the molecular role of ATF2 in these processes is largely unknown. To shed some light on this matter we are identifying ATF2-target genes induced by stimulation of endothelial cells with pro-angiogenic stimuli.

Methods HUVEC were stimulated with various pro-angiogenic stimuli at different times, and the activation (phosphorylation) status of ATF2 was determined by Western Blot.

ATF2 functionality in endothelial cells was suppressed by infecting HUVEC with an adenovirus encoding a phosphorylation-mutant, dominant-negative version of ATF2 (Ad-ATF2AA) where phosphorylation residues Thr69 and Thr71 have been mutated to Ala. HUVEC infection with an adenovirus encoding GFP was used as a control. PCR-based screening of specific gene arrays was used to identify the effect of ATF2 loss- 